\title{
Spububsaúde
}

\section{Enfermagem obstétrica e o parto humanizado}

\author{
Obstetric nursing and humanized delivery

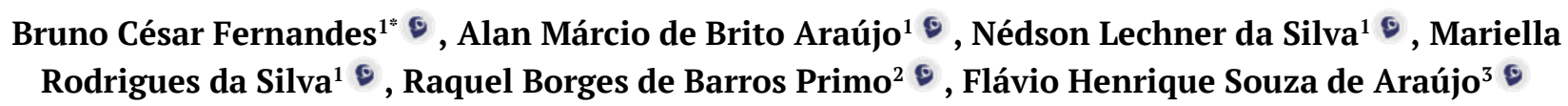

${ }^{1}$ Hospital Universitário da Universidade Federal Grande Dourados, Ebserh, Dourados, Mato Grosso do Sul, Brasil. ${ }^{2}$ Programa de PósGraduação em Ciências da Saúde, Faculdade de Ciências da Saúde, Universidade Federal da Grande Dourados, Dourados, Mato Grosso do Sul, Brasil. *Autor para correspondência. E-mail: brunoanaisafernandes@gmail.com.

\begin{abstract}
Resumo: Introdução: A atenção ao parto vem sofrendo mudanças, se tornando altamente tecnológica. Esse modelo tecnocrático se encontra muito arraigado nas práticas profissionais, valorizando a tecnologia como sinal de sucesso do parto, levando ao uso indiscriminado de práticas intervencionistas desnecessárias, além de um atendimento desumano. Revisão: O objetivo do artigo foi identificar de que maneira a assistência de enfermagem pode auxiliar na valorização do parto humanizado. A realização desse artigo foi por meio de revisão da literatura, sendo pesquisadas informações em programas governamentais de saúde e artigos publicados que abordassem o assunto. Discussão: Foram selecionadas 13 referências para o estudo, sendo nove artigos científicos (dois em inglês e sete em português), dois programas governamentais, um livro e uma publicação da Organização Mundial de Saúde. Considerações finais: Concluiu-se que a humanização do cuidado é fundamental e envolve não apenas a interação do enfermeiro com a parturiente, mas toda uma gama de inter-relações, envolvendo programas de governo voltados para a humanização do cuidado, dedicação à humanização pelos gestores hospitalares, que chegue à equipe de saúde e demais funcionários da instituição, englobando, assim, todo o sistema onde a paciente está inserida.
\end{abstract}

Palavras-chave: enfermagem obstétrica, parto humanizado, violência obstétrica.

\begin{abstract}
Introduction: Childbirth care has undergone changes, becoming highly technological. This technocratic model is very ingrained in professional practices, valuing technology as a sign of successful delivery, leading to the indiscriminate use of unnecessary interventionist practices, in addition to inhumane care. Review: The objective of the article was to identify how nursing care can help to value humanized childbirth. The realization of this article was through a literature review, researching information on government health programs and published articles that addressed the subject. Discussion: 13 references were selected for the study, nine scientific articles (two in English and seven in Portuguese), two government programs, a book and a publication by the World Health Organization. Final remarks: It was concluded that the humanization of care it is fundamental and involves not only the interaction of the nurse with the parturient, but a whole range of interrelationships, involving government programs focused on the humanization of care, dedication to humanization by hospital managers, which reaches the health team and other employees institution, thus encompassing the entire system where the patient is inserted.
\end{abstract}

Keywords: obstetric nursing, humanized birth, obstetric violence.

\section{Introdução}

Nos últimos anos, estudos tem demostrado um problema, crescente e alarmante, que ocorre com mulheres durante o parto, com ou sem complicações, representado por intervenções médicas coonsideradas desnecessárias além de uma disseminação de práticas abusivas e desrespeitosas em relação às parturientes em todo o mundo (Sadler et al., 2016). Existem muitas instituições de saúde, não apenas no Brasil, mas em países do mundo todo, que em prol do uso da tecnologia e devido à falta de humanização do atendimento violam os direitos reprodutivos das mulheres durante o parto, por meio de atitudes que se convertem em formas naturalizadas de violência (Silva \& Serra, 2017).

O reconhecimento dessa realidade fez surgir o termo violência obstétrica, que corresponde a uma forma específica da violência de gênero, já que ocorre uma arbitrariedade do saber por parte de profissionais da saúde no que tange o controle do corpo e da sexualidade das gestantes. Dentro dessa expressão estão reunidas todas as formas de violência e iatrogenias obstétricas, ou seja, enquadram-se nesse conceito todos os atos praticados, seja no corpo da mulher, seja no corpo do neonato, os quais são realizados sem o devido 
consentimento ou informação prestada à paciente (Silva \& Serra, 2017). O termo foi idealizado pelo médico obstetra, Rogelio Pérez D’Gregório e desde então, sua criação tem guiado lutas no movimento feminista em prol da extinção desses atos e da punição daqueles que o cometem (Mariani \& Nascimento Neto, 2016).

A importância da temática foi reconhecida pela Organização Mundial de Saúde (OMS) que no ano de 2014 alertou que a violência obsetrica é um problema da saúde pública impactante na vida de gestantes, puérperas e seus recém-nascidos. No ano anterior, um estudo brasileiro revelou que uma em cada quatro mulheres no país sofre algum tipo de violência durante o parto, sejam eles procedimentos que poderiam ser evitados e que provocam dores na gestante, falta de consentimento da grávida e/ou informação pelo profissional de saúde para a execução de tais procedimentos; carência de ampatia parte dos profissionais de saúde (expressado por gritos), falta de analgesia e até negligência (Venturi Júnior \& Godinho, 2013).

Justifica-se a importância de estudar esse tema devido ao fato de , ainda, existirem serviços de saúde , no Brasil, que extrapolam os direitos reprodutivos das mulheres durante o período do parto e puerpério, permeando a supervalorização da tecnologia em detrimento da humanização do atendimento. A relevância social do tema se justifica pelo fato dessa violência de gênero ainda ser desconhecida pela população. Por outro lado, a relevância profissional do tema se baseia na presença da enfermagem obstétrica, a qual é uma equipe especializada que atua nesse cenário e possui profissionais treinados e capacitados para a realização de uma assistência ao parto humanizado, sendo assim, ideais para evitar que a violência obstétrica aconteça.

Portanto, reforça-se a importância científica e social dessa pesquisa para elucidar como a assistência de enfermagem pode auxiliar no desenvolvimento da cultura e valorização da humanização do parto, evitando então que atos de violência obstétrica ocorram.

Diante do exposto, o objetivo desse artigo foi identificar de que maneira a assistência de enfermagem auxilia na valorização do parto humanizado.

\section{Revisão}

A metodologia escolhida para a realização desse estudo foi a revisão da literatura, com intuito de reunir informações sobre o tema. Foram utilizados livros, programas governamentais da área de saúde e artigos publicados que abordassem o assunto. Os artigos foram pesquisados nos portais de pesquisa Biblioteca Virtual em Saúde (BVS) e no Google Acadêmico, através dos descritores: parto; humanização do parto; humanização na saúde; violência obstétrica; enfermagem; enfermagem obstétrica. Como critérios de inclusão foram selecionados artigos em português e inglês, publicados nos últimos 10 anos e que se encontrassem disponíveis na íntegra online. Os artigos foram analisados e selecionados conforme os critérios de inclusão por meio da leitura de seus títulos e resumos.

Foram selecionadas 13 referências para o estudo, sendo nove artigos científicos (dois em inglês e sete em português), dois programas governamentais, um livro e uma publicação da OMS.

\section{Discussão}

Conforme explicam Mariani e Nascimento Neto (2016), o conceito de violência obstétrica abrange todas as ocasioões onde intervenções de profissionais de saúde são implementados na mão, ou mesmo, no neonato sem o consentimento prévio, além da realização de procedimentos obsoletos, que, no entanto, continuam a ser utilizados. Entre os principais, no cenário do atendimento realizado pelo Sistema Único de Saúde (SUS), estão:

“[...] a episiotomia (corte na região do períneo) e a manobra de Kristeler (quando a barriga é empurrada por enfermeiras), o enema (lavagem intestinal) uso da ocitocina sintética (hormônio acelerador das contrações), da anestesia, do fórceps, o jejum de comida e água, exames de toque frequentes (usados para conferir a dilatação e a descida do bebê), o rompimento artificial da bolsa e a posição horizontal da mulher" (Mariani \& Nascimento Neto, 2016).

Ademais, é importante destacar a relação da violência obstétrica com as variáveis socioeconômicas e demográficas, as quais apresentam grande influência em sua incidência. Tendo isso em vista, determinantes sociais como cor, escolaridade, região e renda têm influência na forma como essas mulheres são tratadas. Cabe ressaltar que essa atitude precisa ser urgentemente revista, já que os profissionais precisam atender às necessidades das parturientes com equidade, dignidade e humanidade (Mariani \& Nascimento Neto, 2016). 
Existem programas do Ministério da Saúde destinados à humanização no atendimento que visam combater a violência obstétrica, tendo como premissa o conceito de atenção humanizada durante o parto, o qual, segundo o Ministério da Saúde (2001) “(...) envolve um conjunto de conhecimentos, práticas e atitudes que visam a promoção do parto e nascimento saudáveis e a prevenção da morbimortalidade materna e perinatal”. Para isso, foi desenvolvido o Programa de Humanização no Pré-Natal e Nascimento (PHPN), em 2000, que objetivava, primordialmente, (Brasil, 2002) "assegurar a melhoria do acesso, da cobertura e da qualidade do acompanhamento pré-natal, da assistência ao parto e puerpério às gestantes e ao recémnascido, na perspectiva dos direitos de cidadania”-(Brasil, 2001).

As diretrizes preconizadas pelo PHPN determinam práticas para receber a parturiente de forma cordial e respeitosa, se responsabilizando por essa mulher integralmente, sem ditar regras, ouvindo suas reclamações e permitindo que ela possa expor suas preocupações e dúvidas. Deve, também, prestar orientações a família, de forma a garantir a conquista da continuidade dos serviços de saúde (Moura et al., 2018). Posteriormente, foi instituída em 2003, a Política Nacional de Humanização (PNH), com o objetivo de efetivar os princípios do SUS no cotidiano das práticas de atenção e de gestão, e também estimular trocas solidárias entre gestores, trabalhadores e usuários para a produção de saúde (Brasil, 2013).

Segundo Andrade e Aggio (2014), a humanização do parto se traduz na necessidade de modificações a respeito da compreensão geral do parto como experiência humana e, além disso, para os profissionais que prestam o atendimento, representa uma transformação em como agir no momento oportuno perante o sofrimento do próximo. Ademais, Alvares et al. (2018) defendem que é essencial que a gestante possua informações sobre seus direitos e quais práticas assistenciais são ou não benéficas para ela e seu recémnascido, tendo consciência sobre como se dá um cuidado humanizado ao parto, pois só assim ela conseguirá ser protagonista e ter sua autonomia respeitada no momento do nascimento.

Um dos fatores que mais traz influência positiva para a satisfação materna é o modo como a gestante é tratada e acolhida pelos profissionais que a recebem e atendem, visto que eles amenizam a ansiedade vivida pela mulher durante todo o processo de parturição (Alvares et al., 2018). Para atuar nesse atendimento, a enfermagem obstétrica, composta por profissionais treinados e aptos para realizar uma assistência ao parto mais humanizada, tem o objetivo de proporcionar às mães maior conforto e segurança, por meio da escuta ativa e do fornecimento de informações.

Estes profissionais têm demonstrado sua importância, não apenas para a humanização do parto, mas também para sua desmedicalização, pois têm a competência de selecionar práticas que não interfiram na fisiologia do parto, com o intuito de tornar a gestante e seu(s) acompanhante(s) mais ativo(s) e participativo(s) (Alvares et al., 2018).

Uma questão importante, do ponto de vista dos profissionais enfermeiros, é trazida pelo estudo de Villa e Flores (2018), em que as autoras tiveram como objetivo identificar as experiências profissionais que influenciam no processo de significação do cuidado obstétrico. Verificou-se que é um grande desafio viver e trabalhar com profissionais que, por questões associadas à diferença entre gerações, entre outros motivos, divergem nos valores relacionados ao cuidado. Isso se deve a modificações nos perfis dos programas acadêmicos que compõem a formação do aluno como recurso humano na saúde.

Nesse sentido, as autoras ressaltam que a formação da equipe médica geralmente é feita sob uma perspectiva individualizada e técnica em detrimento de uma abordagem ética e humanizada. Dessa maneira, muitas vezes a relação de trabalho médico-enfermeiro é afetada por comportamentos hierarquizantes e hostis, o que dificulta a excelência do cuidado com o paciente. Além disso, as profissionais enfermeiras obstétricas entrevistadas apontam para a corresponsabilidade das instituições formadoras de recursos humanos pela crise de valores identificada e indicam que é necessário reajustar os programas para aprimorar as habilidades das novas gerações em termos de assistência humanitária Villa e Flores (2018).

Moura et al. (2018) estabeleceram sete medidas de prevenção à violência obstétrica que devem ser realizadas pela equipe de enfermagem, a fim de realizar boas práticas e prevenir sua ocorrência:

“[...] Explicar para a paciente de maneira que ela compreenda o que ela tem, o que pode ser feito por ela e como ela pode ajudar. 2- Evitar procedimentos invasivos, que causem dor e que sejam arriscados, exceto em situações estritamente indicadas; 3 - Procurar ouvir a paciente e trabalhar em parceria com os colegas e garantir um tratamento ao paciente longe do humilhante; 4Promover a paciente o direito de acompanhante de sua escolha no pré-natal e parto; 5- Garantir o acesso ao leito e uma assistência pautada na equidade; 6Orientar a mulher acerca dos direitos relacionados a maternidade e reprodução; 7 - 
Investir em si mesmo, buscando realização no seu trabalho e estar em constante atualização" (Moura et al., 2018).

Uma questão importante no cenário da obstetrícia é que esses profissionais devem garantir a todas as parturientes acesso a informações imparciais e baseadas em evidências sobre as intervenções que lhes forem sugeridas (Sadler et al., 2016). Pesquisas desenvolvidas por Romão et al. (2018) esclarecem que algumas atitudes devem ser priorizadas por esses profissionais na assistência ao parto normal, como a diminuição da divergência de condutas, a redução de intervenções desnecessárias e a implementação das práticas baseadas em evidências.

Nos estudos desenvolvidos por Villa e Flores (2018) as enfermeiras entrevistadas durante a pesquisa também alertam para outro fato importante - a questão da abordagem preventiva, precária em muitos lugares na atenção básica. Tal aspecto repercute no fato de as mulheres chegarem às instituições com alta incidência de complicações e sem saber como participar mais ativamente de seus partos. Esses são ensinamentos que deveriam ter sido fornecidos nas consultas pré-natais, as quais também constituem o trabalho do enfermeiro obstétrico.

Diante de ações humanizadas na assistência ao parto, verifica-se que há uma grande influência destas na qualidade da evolução do parto e na saúde da mãe e seu filho, o que proporciona redução e otimização dos custos e queda nas taxas de mortalidade (Romão et al., 2018).

Tal fato é corroborado pelo estudo de Medeiros et al. (2016), que teve por objetivo analisar a assistência obstétrica prestada em uma unidade de Pré-parto/Parto/Pós-parto (PPP) em um hospital de ensino no estado de Mato Grosso depois da inclusão da enfermagem obstétrica. Os resultados sugerem que essa inserção contribuiu para a qualificação do cuidado prestado ao parto e ao nascimento, o que foi evidenciado pela redução de intervenções, como a episiotomia e as cesarianas. Além disso, houve incentivo ao uso de práticas que não interferem na fisiologia do processo parturitivo, gerando bons resultados perinatais (Medeiros et al., 2016).

Diante disso, torna-se necessário que mudanças preventivas sejam realizadas objetivando promover a humanização, o que deve ocorrer desde a formação desses profissionais. Sendo assim, o enfermeiro obstetra deve agir buscando respeitar os sentimentos e emoções da parturiente, de maneira a não desvalorizá-los no momento do parto. As organizações de saúde, principalmente na figura do enfermeiro, devem priorizar o acolhimento da mulher e sua família de forma digna para criar um ambiente que garanta à paciente sua autonomia. Ainda, a equipe de enfermagem deve assegurar um atendimento adequado, bem como permitir que a gestante conheça o local em que realizará seu parto, garantindo a ela seu atendimento humanizado de direito em todas as etapas da gravidez (Moura et al., 2018).

\section{Considerações finais}

A violência obstétrica ainda é pouco discutida pela população, mas se encontra muito presente na rotina das maternidades brasileiras, especialmente nas públicas, relacionada à falta de humanização no cuidado que implica em assistência, praticada por profissionais de saúde, permeada por agressões de natureza física, mental e verbal contra as mulheres.

Dessa maneira, a humanização do cuidado é fundamental e envolve não apenas a interação do cuidador com a parturiente, mas também toda uma gama de interrelações entre equipe de saúde e demais funcionários da instituição, englobando, assim, todo o sistema onde a paciente está inserida. Além disso, vale destacar a importância dos programas do governo no incentivo à prática da humanização do cuidado em saúde.

Nesse cenário, destacam-se os profissionais enfermeiros obstétricos, que são peça-chave no processo de humanização do atendimento, já que passam a maior parte do tempo com a gestante e sua família e/ou acompanhante(s). Assim, constata-se que a assistência de enfermagem é fundamental para a valorização do parto humanizado, pois esses profissionais acompanham todas as etapas do parto, inclusive o pré-natal, período em que diversas informações são concedidas às gestantes.

Cabe destacar a importância da formação desses profissionais, tendo em vista que é o momento no qual os valores são lapidados. Assim, uma gestão hospitalar humanizada enfrentará menos empecilhos para ser implementada por não haver resistência cultural dos atores envolvidos.

Conclui-se que, no nível educacional, os princípios dos direitos humanos no parto e a discussão sobre violência obstétrica devem ser incluídos no currículo de diversas instituições educacionais, enfatizando suas 
dimensões relacionadas ao gênero. Dessa forma, a violência obstétrica não mais será silenciada nem normalizada, e as mulheres passarão a exercer, com propriedade, seus direitos.

\section{Referências}

Alvares, A. S., Corrêa, A. C. P., Nakagawa, J. T. T., Teixeira, R. C., Nicolini, A.B. \& Medeiros, R. M. K. 2018. Práticas humanizadas da enfermeira obstétrica: contribuições no bem-estar materno. Revista Brasileira de Enfermagem, 71(supl.6), 2620-2627.

Andrade, B. P. \& Aggio, C. M. 2014. Violência obstétrica: a dor que cala. Anais do III Simpósio Gênero e Políticas Públicas. Universidade Estadual de Londrina, 27 a 29 mai. Disponível em: http:// encurtador.com.br/emDF8. Acesso em: 06 dez. 2020.

Brasil, Ministério da Saúde. 2001. Parto, aborto e puerpério: assistência humanizada à mulher. Brasília: Ministério da Saúde. Disponível em: https://bvsms.saude.gov.br/bvs/publicacoes/cd04_13.pdf. Acesso em: 06 dez. 2020.

Brasil, Ministério da Saúde. 2002. Programa Humanização do Parto, Humanização no pré-natal e nascimento. Brasília, DF: Ministério da Saúde. Disponível em: https:// https://bvsms.saude.gov.br/bvs/publicacoes/parto.pdf. Acesso em: $06 \mathrm{dez}$.

Brasil, Ministério da Saúde. 2013. Política Nacional de Humanização - PNH. Brasília, DF: Ministério da Saúde Disponível

em: http://bvsms.saude.gov.br/bvs/publicacoes/politica_nacional_humanizacao_pnh_folheto.pdf. Acesso em: 06 dez. 2020.

Mariani, A. C. \& Nascimento Neto, J. O. 2016. Violência obstétrica como violência de gênero e violência institucionalizada: breves considerações a partir dos direitos humanos e do respeito às mulheres. Cadernos da Escola de Direito, 2(25), 48-60.

Medeiros, R. M. K., Teixeira, R. C., Nicolini, A. B., Alvares, A. S., Corrêa, A. C. P., \& Martins, D. P. 2016. Cuidados humanizados: a inserção de enfermeiras obstétricas em um hospital de ensino. Revista Brasileira de Enfermagem,69(6), 1091-1098.

Moura, R. C. D. M., Pereira, T. F., Rebouças, F. J., Costa, C. D. M., Lernades, A. M. G., Silva, L. K. A. D., \& Rocha, K. D. M. M. D. 2018. Cuidados de enfermagem na prevenção da violência obstétrica. Enfermagem em foco, 60-65.

Organização Mundial Da Saúde [OMS]. 2014. Prevenção e eliminação de abusos, desrespeito e maus-tratos durante o parto em instituições de saúde. Genebra, $\mathrm{CH}$ : Departamento de Saúde Disponível em: https://apps.who.int/iris/bitstream/handle/10665/134588/WHO_RHR_14.23_por.pdf;jsessionid=4CAE20 66ECE2C5550A7D7B87336E970D?sequence=3. Acesso em: 06 dez. 2020.

Romão, R. S., Fuzissaki, M. A., Prudêncio, P. S., \& Freitas, E. A. M. 2018. Qualidade da assistência obstétrica relacionada ao parto por via vaginal: estudo transversal. Revista de Enfermagem do Centro Oeste Mineiro, 8, e2907.

Sadler, M., Santos, M. J., Ruiz-Berdún, D., Rojas, G. L., Skoko, E., Gillen, P., \& Clausen, J. A. 2016. Moving beyond disrespect and abuse: addressing the structural dimensions of obstetric violence. Reproductive Health Matters, 24(47), 47-55.

Silva, A. S. E. \& Serra, M. C. M. 2017. Violência obstétrica no Brasil: um enfoque a partir dos acórdãos do STF e STJ. Quaestio Iuris, 10(4), 2430-2457.

Venturi Júnior, G., \& Godinho, T. 2013. Mulheres brasileiras e gênero nos espaços público e privado: uma década de mudanças na opinião pública. São Paulo, SP: Fundação Perseu Abramo.

Villa, C. M., \& Flores, Y. R. 2018. Experiences influencing upon the significance of obstetric care in mexican nurses. Investigación y Educación en Enfermería, 36(1), e12.

\section{Minicurrículo}

Bruno César Fernandes. Enfermeiro Assistêncial da Empresa Brasileira de Serviços Hospirtalares (EBERH/HU-UFGD), mestrando do Programa de Pós-Graduação Stricto Sensu Mestrado Profissional Ensino em Saúde da Universidade Estadual do Mato Grosso do Sul (PPGES-UEMS) polo Dourados/MS. Pós-graduado 
em Unidade de Terapia Intensiva, Unidade de Terapia Intensiva Neonatal e Pediátrica (Ingá) e, em Saúde da Família.

Alan Márcio de Brito Araújo. Enfermeiro Assistêncial da Empresa Brasileira de Serviços Hospirtalares (EBERH/HU-UFGD). Especialização em Saúde da Família, Terapia Intensiva Adulto, e, Residência Multiprofissional em Neurologia.

Nédson Lechner da Silva. Possui graduação em Negócios Imobiliários e graduação em Enfermagem. Atualmente é Funcionário Público da EBSERH HU/UFGD, ocupando o cargo de Enfermeiro Assistencial, lotado na UTI Adulto. Especialização em Práticas Avançadas de Enfermagem na Atenção Primária.

Mariella Rodrigues da Silva. Enfermeiro Assistêncial da Empresa Brasileira de Serviços Hospirtalares (EBERH/HU-UFGD).

Raquel Borges de Barros Primo. Doutoranda em Ciências da Saúde pela Universidade Federal da Grande Dourados. Mestre em Ciências da Saúde pela Universidade Federal da Grande Dourados. Graduada em Enfermagem. Atualmente é enfermeira assitencial - EBSERH HUPES-UFBA.

Raquel Borges de Barros Primo. Graduado em Ciências Biológicas e Educação Física Bacharelado. Atualmente é Doutorando no Programa de Pós-graduação em Ciências da Saúde na Faculdade de Ciências da Saúde na Universidade Federal da Grande Dourados (FCS/UFGD).

Como citar: Fernandes, B.C., Araújo, A.M.B., Silva, N.L., Silva, M.R., Primo, R.B.B., \& Araújo, F.H.S. 2021. Enfermagem obstétrica e o parto humanizado, 6, 134. DOI: https://dx.doi.org/10.31533/pubsaude6.a134

Recebido: 12 fev. 2021.

Revisado e aceito: 1 mar. 2021.

Conflito de interesse: os autores declaram, em relação aos produtos e companhias descritos nesse artigo, não ter interesses associativos, comerciais, de propriedade ou financeiros que representem conflito de interesse.

Licenciamento: Este artigo é publicado na modalidade Acesso Aberto sob a licença Creative Commons Atribuição 4.0 (CC-BY 4.0). 